\title{
SUR LA PONTE DU GASTROPHILUS PECORUM
}

\author{
Par G. DiNULESCU
}

Toutes les recherches faites jusqu’à présent sur la biologie du Gastrophilus pecorum ne disent rien sur les phénomènes de la ponte et le lieu où sont déposés les œufs de cette espèce. Seul - Hobmeyer établit une relation entre la couleur des œufs et le lieu de la ponte et, sans aucune observation, il suppose que la femelle doit déposer'ses œufs sur la terre. En fait, aucun auteur n’a précisé l'endroit où sont déposés les œufs.

Observations personnelles. - Le Gastrophilus pecorum étant fréquent en Roumanie, j’ai cherché sur des centaines de chevaux, de préférence sur les animaux à poils blanes, les œufs noirs de G. pecorum mais, moi aussi, je n’ai réussi à les trouver sur aucun animal. Pourtant, d'après une observation faite au laboratoire, je crois pouvoir donner des indications précises sur le lieu de la ponte des œufs de G. pecorum.

J'ai mis, le 2 septembre 1927, un lot de 14 larves de G. pecorum au dernier stade, dans un des bocaux contenant des élevages des différentes espèces de larves de Gastrophilus. Le bocal était couvert avec un morceau de gaze. Pendant la période de sortie des mouches, le lot était examiné chaque jour.

Au bout de 21 jours, les mouches ont commencé à sortir. Du $21^{\circ}$ jour au $27^{\circ}$ jour, sont sortis 5 mâles et 6 femelles. Après 35 jours, les trois autres larves se sont transformées en nymphes qui sont mortes sans éclore.

Au cours du mois de mai de cette année (1929), en examinant de nouveau mon matériel de Gastrophilus, j'ai pu observer sur deux de ces trois nymphes des petits groupes d'œufs de Gastrophilus pecorum. Sur une nymphe, il y avait 16 œufs (fig. 1) et sur l'autre 2 œufs. J'ai observé encore sur la calotte d'une des dépouilles nymphales un groupe de huit œufs. Deux d'entre eux étaient éclos car leurs coques étaient désoperculées. Ces œufs sont collés par leur extrémité postérieure sur la coque de la nymphe et ils sont fixés en petits amas comme on le voit sur la figure 1. L'appendice fibro-glutineux qui prolonge les œufs à leur extrémité opposée à l'opercule s'étale en éventail sur la surface de la coque de la nymphe.

Annales de Parasitologie, $\mathrm{T}$. VII, $\mathrm{N}^{\circ} 4 .-1^{\text {er }}$ juillet 1929 , p. 287-289. 
Je donne ici la description de la morphologie extérieure de l'œuf de Gastrophilus pecorum :

a. Euf extrait de l'utérus. - Couleur : noir brillant, forme allongée, faiblement elliptique et légèrement aplatie latéralement. L'œuf est pointu

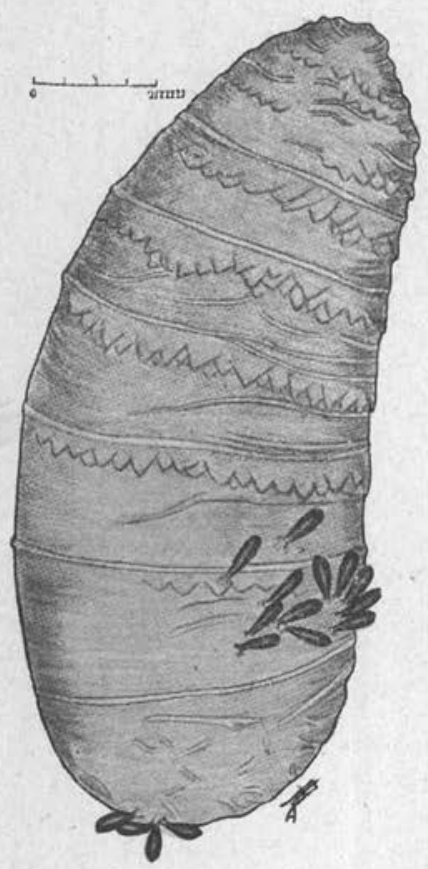

Fig. 1. - Nymphe de Gastrophilus pecorum à la surface de laquelle on voit deux groupes d'œufs de. G. pecorum.

à une extrémité, il est tronqué et operculé à l'extrémité opposée. L'œuf est prolongé par un petit amas globuleux de substance glutineuse ; son extrémité inférieure pointue pénètre jusqu'à la moitié de cet amas ( $a$, fig. 2). Dimensions : longueur $860 \mu$, largeur au niveau de l'opercule $260 \mu$ de face et $220 \mu$ de côté. Le diamètre du renflement glutineux varie . de 120 à $150 \mu$.

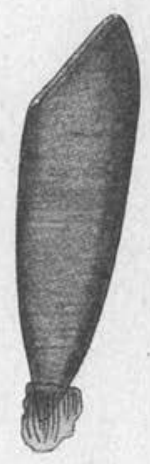

a.

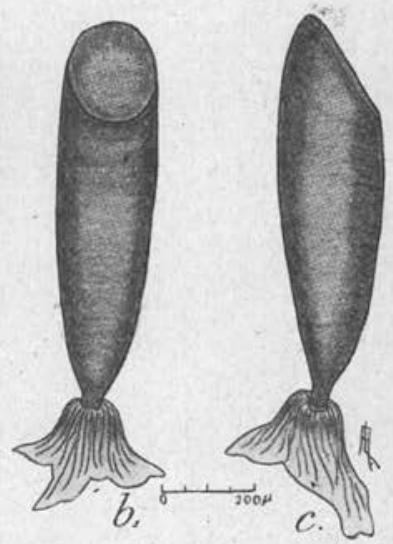

Fıg. 2. $-a$, Euf extrait de l'utérus d'une femelle de Gastrophilus pecorum; $b$, wuf pondu, vu de face; $c$, le même vu de profil.

b. Euf pondu détaché de la coque nymphale. - Même couleur et même forme. A son extrémité pointue, l'œuf se détache un peu de la portion glutineuse et semble attaché par un petit cordon court. La portion glutineuse prend ici la forme d'un cône tronqué à l'endroit où elle est attachée à l'œuf. La base de ce cône, c'est-à-dire la base d'attachement, s'étale en éventail à la surface du support ( $b$ et $c$, fig. 2).

Dimensions : longueur $840 \mu$, largeur au niveau de l'opercule $240 \mu$ de face et $220 \mu$ de côté. La substance glutineuse a une hauteur de $150 \mu$ et à la base un diamètre de $400 \mu$.

Quand les œufs sont déposés par groupes, les masses glutineuses confluent ; les œufs sont implantés obliquement et convergent plus ou moins par leur extrémité inférieure. 
De cette observation on peut déduire que la femelle de Gastrophilus pecorum dépose ses œufs sur le fourrage ou les objets qui se trouvent dans les écuries ou au voisinage des chevaux, de sorte que les jeunes larves peuvent être introduites par la bouche dans le tube digestif du cheval.

J'ai compté sur trois femelles de Gastrophilus pecorum le nombre des oufs extraits de leur utérus, j’ai trouvé des chiffres variant entre 2343 et 2560 . On voit que le nombre des œufs de cette espèce est bien supérieur à celui des oufs des autres espèces qui ont la chance de déposer leurs œufs sur l'animal lui-même et quelquefois tout à fait près de la bouche, là où ils ont le plus de chances d'arriver directement dans le tube digestif. Le grand nombre des œufs de $G$. pecorum compense les risques auxquels sont exposées les jeunes larves sorties de ces œufs dispersés partout et quelquefois loin de leur hôte.

\section{RÉsumé}

Jusqu'ici on ne connaissait pas le lieu de la ponte du Gastrophilus pecorum, et je l'ai moi-même cherché en vain sur les chevaux. J'ai eu la bonne fortune de trouver ces pontes sur des nymphes de la même espèce placées dans des bocaux d'élevage. Je me crois autorisé à en conclure que les femelles de Gastrophilus pecorum peuvent pondre sur des objets quelconques au voisinage des animaux et non sur les animaux eux-mêmes.

\section{BibliographiE}

HовметвR- (M.). - Beiträge zur Biologie und Bekämpfung der Gastrus Fliege (Gastrophilus equî). Monatshefte fur praktische Tierheilkunde, XXIX, 1917, p. 153.

Dove (W.-E.). - Some biological and control Studies of Gastrophilus hrmorroidalis and other bots of horses. United States Department of Agriculture, Bull. n 597, 1918, p. 27, 30 et 33.

Laboraloires de Parasitologie de la Facullé de Médecine Vétérinaire de Bucarest el de la Faculté de Médecine de Paris. 